\title{
The impact of unilateral versus bilateral antegrade cerebral perfusion on surgical outcomes after aortic arch replacement: A propensity-matched analysis
}

\author{
Andreas Zierer, MD, ${ }^{a}$ Petar Risteski, MD, ${ }^{\text {a }}$ Ali El-Sayed Ahmad, MD, ${ }^{\text {a }}$ Anton Moritz, MD, ${ }^{\text {a }}$ \\ Anno Diegeler, MD, ${ }^{b}$ and Paul P. Urbanski, MD $^{\mathrm{b}}$
}

\begin{abstract}
Objectives: Antegrade cerebral perfusion during mild systemic hypothermia has been successfully used in aortic arch surgery. Whether bilateral antegrade cerebral perfusion is more neuroprotective then unilateral antegrade cerebral perfusion has been the subject of much debate.
\end{abstract}

\begin{abstract}
Methods: Between 2000 and 2012, 1097 patients underwent aortic arch replacement using $28^{\circ} \mathrm{C}$ to $30^{\circ} \mathrm{C}$ antegrade cerebral perfusion perfusate during mild systemic hypothermia in a pressure-controlled manner $(\geq 70 \mathrm{~mm} \mathrm{Hg}$ ). After excluding acute type A dissections, 738 elective surgeries with unilateral $(\mathrm{n}=477)$ or bilateral $(n=261)$ antegrade cerebral perfusion underwent logistic regression analysis with covariates among 9 patient variables (age, coronary disease, diabetes, renal failure, stroke, antegrade cerebral perfusion time, partial vs total arch replacement, aortic valve replacement, and cardiopulmonary bypass time). By using the significant regression coefficients, each patient's propensity score was calculated, allowing selectively matched subgroups of 246 patients each. Operative outcomes were analyzed for differences.
\end{abstract}

Results: There was no significant difference between unilateral and bilateral antegrade cerebral perfusion groups in early mortality $(9 / 246: 4 \%$ vs $11 / 246: 4 \% ; P=.8)$ and transient neurologic deficits $(12 / 246: 5 \%$ vs $10 / 246: 4 \% ; P=.8)$. A trend toward a higher incidence of stroke with bilateral antegrade cerebral perfusion $(5 / 246: 2 \%$ vs $14 / 246: 6 \% ; P=.06)$ was observed. Similar results regarding transient neurologic deficits $(6 /$ $91: 7 \%$ vs $5 / 93: 5 \% ; P=.7)$ and the incidence of stroke $(2 / 91: 3 \%$ vs $9 / 93: 9 \% ; P=.06)$ were observed in matched patients with total arch replacement only.

Conclusions: Using unilateral antegrade cerebral perfusion in a pressure-controlled manner during mild systemic hypothermia is a safe protection strategy in elective aortic arch surgery, associated with similar morbidity and mortality in comparison with bilateral antegrade cerebral perfusion, even if total arch replacement is required. Bilateral antegrade cerebral perfusion reveals a trend of higher incidence of stroke, probably due to manipulation on the arch vessels. (J Thorac Cardiovasc Surg 2014;147:1212-8)

Cerebral protection during aortic arch surgery is still a controversial issue. Deep hypothermic circulatory arrest has been traditionally seen as the mainstay of brain protection during operations on the aortic arch. ${ }^{1}$ To extend the safe period of circulatory arrest and avoid its well-documented drawbacks, antegrade cerebral perfusion (ACP) was

From the Division of Thoracic and Cardiovascular Surgery, ${ }^{\text {a }}$ Johann-WolfgangGoethe University Frankfurt/Main, Frankfurt Main, Germany; and Cardiovascular Clinic Bad Neustadt, ${ }^{\mathrm{b}}$ Bad Neustadt, Germany.

Disclosures: Dr Urbanski discloses a financial relationship with InterVascular, and MAQUET Cardiovascular, Inc. All other authors have nothing to disclose with regard to commercial support.

Drs Zierer and Risteski contributed equally to the study.

Read at the 93rd Annual Meeting of The American Association for Thoracic Surgery, Minneapolis, Minnesota, May 4-8, 2013.

Received for publication April 22, 2013; revisions received Oct 4, 2013; accepted for publication Dec 4, 2013; available ahead of print Feb 7, 2014.

Address for reprints: Andreas Zierer, MD, Division of Thoracic and Cardiovascular Surgery, Johann Wolfgang Goethe University, Theodor Stern Kai 7, 60590 Frankfurt am Main, Germany (E-mail: andreas.zierer@kgu.de).

$0022-5223 / \$ 36.00$

Copyright $(c) 2014$ by The American Association for Thoracic Surgery

http://dx.doi.org/10.1016/j.jtcvs.2013.12.022 introduced. ${ }^{2,3}$ Crittenden and colleagues ${ }^{4}$ were the first to demonstrate experimentally in sheep the clear superiority of ACP over any other method of cerebral protection. Bachet and colleagues ${ }^{5}$ and Kazui and colleagues ${ }^{6}$ were among the early clinical pioneers of cold ACP with deep hypothermic circulatory arrest as method of choice for cerebral protection in the 1990s.

Approximately 1 decade ago, the absolute necessity for deep hypothermia during aortic arch surgery, once ACP with flow rates and pressures within the physiologic range is provided, has been questioned by several institutions. . $^{7-9}$ Different groups now reporting on mortality and neurologic morbidity after aortic arch surgery using mild systemic hypothermia (MSH) in conjunction with ACP often rely on unilateral antegrade cerebral perfusion (UACP). ${ }^{7-10}$ UACP is rapidly installed, avoids manipulation of the supra-aortic vessels, and provides freedom of interruption of cerebral perfusion during aortic arch replacement. However, a competent and effective cerebral collateral network is mandatory if cerebral protection is performed with UACP. Concerns apart of the completeness 


\section{Abbreviations and Acronyms \\ $\mathrm{ACP}=$ antegrade cerebral perfusion \\ $\mathrm{BACP}=$ bilateral antegrade cerebral perfusion \\ $\mathrm{CPB}=$ cardiopulmonary bypass \\ MSH = mild systemic hypothermia \\ NIRS $=$ near-infrared spectroscopy \\ PND = permanent neurologic deficit \\ TND $=$ temporary neurologic dysfunction \\ $\mathrm{UACP}=$ unilateral antegrade cerebral perfusion}

of the cerebral protection also arise in regard to cerebral hyperperfusion, causing brain tissue edema when perfusing the brain from only 1 side. In the absence of scientific evidence, criticism of UACP has been intuitively favoring bilateral antegrade cerebral perfusion (BACP) as a more physiologic and therefore safer method of cerebral protection. Thus, the purpose of the current study was to analyze whether BACP is really more effective then UACP when used for cerebral protection during elective aortic arch operations in MSH.

\section{MATERIALS AND METHODS}

Between 2000 and 2012, 1097 consecutive patients underwent aortic arch repair during $\mathrm{ACP}$ with $\mathrm{MSH}\left(30^{\circ} \mathrm{C} \pm 2{ }^{\circ} \mathrm{C}\right.$; range, $\left.26^{\circ} \mathrm{C}-34^{\circ} \mathrm{C}\right)$ at the Division of Thoracic and Cardiovascular Surgery, Johann Wolfgang Goethe University in Frankfurt am Main and the Cardiovascular Clinic in Bad Neustadt, both in Germany. After excluding acute type A dissections, 738 patients with elective surgery using UACP $(n=477)$ or BACP $(\mathrm{n}=261)$ underwent logistic regression analysis with covariates among 9 patient variables (Table 1). By using the significant regression coefficients, each patient's propensity score was calculated, allowing selectively matched subgroups of 246 patients each. The mean age of patients was 66 \pm 9 years in the UACP group and $65 \pm 11$ years in the BACP group. There were 164 men $(67 \%)$ in the UACP group and 156 men $(63 \%)$ in the BACP group. Detailed preoperative characteristics of propensity-matched patients are listed in Table 2 . The study was approved by the 2 institutional review boards, and individual patient consent for the study was waived.

\section{Operative Techniques}

Surgical protocols applied in Frankfurt and Bad Neustadt have been described in detail. ${ }^{9,11-14}$ Briefly, treatment in Frankfurt included cannulation of the left radial artery and one of the femoral arteries for continuous blood pressure monitoring. The right axillary artery was exposed and directly cannulated with an $18 \mathrm{~F}$ to $22 \mathrm{~F}$ flexible arterial cannula (Edwards Lifesciences, Irvine, Calif) followed by cannulation of the right atrium in a standard fashion. Cardiopulmonary bypass (CPB) was started, and cooling was limited to $30^{\circ} \mathrm{C} \pm 2{ }^{\circ} \mathrm{C}$ rectal temperature, depending on the expected time of arch repair according to the findings on the preoperative computed tomography scan. The innominate and left carotid artery were snared with silicone elastomer loops and occluded at the initiation of selective ACP. After opening the aortic arch, the left subclavian artery was blocked by insertion of a Fogarty catheter to obtain a bloodless operative field. In patients with BACP, the elastomer loop snared around the left common carotid artery was temporarily loosened at this point, and a Y-shaped arterial line connected to the arterial CPB cannula was placed inside the vessel for additional perfusion of the left hemisphere. BACP was the method of choice between 2005 and 2010. Before 2005 and after 2010, there was a trend toward UACP. Selective ACP was conducted with a perfusate temperature of $28^{\circ} \mathrm{C}$ to $30^{\circ} \mathrm{C}$ in a pressure-controlled manner. The perfusion pressure was controlled on the pump unit and kept at $75 \mathrm{~mm} \mathrm{Hg}$, allowing for a UACP flow of $1.1 \pm 0.2 \mathrm{~L} / \mathrm{min}$ and a respective BACP flow of $1.4 \pm 0.3 \mathrm{~L} / \mathrm{min}$. At that point, the arch replacement was performed, including a reimplantation of the arch vessels en bloc if necessary, followed by reconstitution of full body perfusion by way of the right axillary artery once the arch replacement was completed. Neurovascular monitoring varied throughout the study period and consisted of cerebral saturation assessment using near-infrared spectroscopy (NIRS) in the latter half of the series.

In Bad Neustadt, the arterial return for $\mathrm{CPB}$ was achieved by isolated cannulation of the right or left or both common carotid arteries in the majority of patients. Alternative cannulation of the innominate artery was considered in selected patients. During ACP, a Y-shaped line connected to the arterial return cannula for $\mathrm{CPB}$ was used to perfuse the other brain hemisphere in patients undergoing operation with BACP. The decision to choose the particular cannulation technique was made preoperatively according to the findings from computed tomography. The specific cannulation technique has been described in detail. ${ }^{13,14}$ In all patients, after heparinization, the exposed segment of the according artery was crossclamped and an 8- or 10-mm vascular sealed polyester graft was anastomosed to the artery. Systemic circulatory arrest during selective ACP was used for arch replacement. Neurovascular monitoring varied during the study period and currently consists of pressure measurement in both radial arteries and cerebral saturation assessment using NIRS. The deepest temperature was determined by the surgeon in accordance with the expected period of circulatory arrest and averaged $30^{\circ} \mathrm{C} \pm 2^{\circ} \mathrm{C}$ rectal temperature. ACP was always performed at an arterial blood temperature of $28^{\circ} \mathrm{C}$. The arch arteries were crossclamped at the beginning of ACP. UACP was set at a perfusion pressure on the pump unit of $80 \mathrm{~mm} \mathrm{Hg}$, allowing for a UACP flow of $0.9 \pm 0.2 \mathrm{~L} / \mathrm{min}$ when the left common carotid artery was used for perfusion and, considering the flow to the right arm, a flow of $1.5 \pm 0.3 \mathrm{~L} / \mathrm{min}$ when the innominate artery or the right common carotid artery was used. During rewarming, the Y-shaped arterial line was used to switch the arterial perfusion from the cannulated artery to the aortic graft. Isolated anastomosing of supra-aortic vessels with respective graft branches is the preferred technique for total arch replacement in Bad Neustadt, for which vascular prostheses with 4 side branches (InterGard AorticArch; InterVascular, La Ciotat, France) are used. In both institutions, during rewarming, the temperature gradient between the oxygenated blood (arterial line) and the patient's core temperature was set at a maximum of $10^{\circ} \mathrm{C}$, with a peak temperature of blood leaving the oxygenator of $38.5^{\circ} \mathrm{C}$.

\section{Statistical Analysis}

All perioperative data were collected prospectively. Study end points were operative mortality and neurologic morbidity. Temporary neurologic dysfunction (TND) was defined as the presence of reversible postoperative motor deficit, confusion, agitation, or transient delirium. Computed tomography findings were required to be normal, with resolution of all symptoms before discharge. Permanent neurologic deficit (PND) was defined as the presence of new focal (stroke) or global (coma) permanent neurologic dysfunction. Early mortality was defined as death during the initial hospitalization or within 30 days postoperatively for discharged patients. Continuous data are reported as mean \pm standard deviation. The Fisher exact test was used for categoric variables, and paired, 2-tailed $t$ test was used for continuous data. The nonrandomness of procedure assignment was addressed by propensity matching to provide a more reliable assessment of outcomes based on procedure type. Logistic regression analysis was used to identify covariates among 9 baseline patient variables that were imbalanced in the 2 groups of interest. Variables included age, coronary disease, diabetes, renal failure, stroke, ACP time, partial versus total arch replacement, aortic valve replacement, and CPB time. The total population 
TABLE 1. Propensity-matched preoperative and perioperative variables of patients undergoing aortic arch replacement during selective unilateral or bilateral antegrade cerebral perfusion and mild systemic hypothermic circulatory arrest

\begin{tabular}{lccr}
\hline \multicolumn{1}{c}{ Characteristic } & UACP & BACP & $\boldsymbol{P}$ value \\
\hline Age $(\mathrm{y} \pm \mathrm{SD})$ & $66 \pm 9$ & $65 \pm 11$ & $>.99$ \\
Coronary artery disease (N/\%) & $39(16 \%)$ & $42(17 \%)$ & .68 \\
Diabetes (N/\%) & $20(8 \%)$ & $18(7 \%)$ & .65 \\
Chronic renal failure* (N/\%) & $13(5 \%)$ & $13(5 \%)$ & $>.99$ \\
Previous stroke (N/\%) & $22(9 \%)$ & $20(8 \%)$ & .73 \\
Selective ACP time (min) & $41 \pm 19$ & $43 \pm 21$ & $>.99$ \\
Hemi-arch replacement (N/\%) & $155(63 \%)$ & $153(62 \%)$ & .59 \\
Total arch replacement (N/\%) & $91(37 \%)$ & $93(38 \%)$ & .71 \\
Concomitant AVS (N/\%) & $79(32 \%)$ & $71(29 \%)$ & .69 \\
CPB time (min) & $164 \pm 41$ & $157 \pm 37$ & .81 \\
\hline
\end{tabular}

$A C P$, Antegrade cerebral perfusion; $A V S$, aortic valve surgery (including valve repair replacement and conduit); $B A C P$, bilateral antegrade cerebral perfusion; $C P B$, cardiopulmonary bypass; $S D$, standard deviation; $U A C P$, unilateral antegrade cerebral perfusion. $*$ Chronic renal failure $=$ glomerular filtration rate $<60 \mathrm{~mL} / \mathrm{min} / 1.73 \mathrm{~m}^{2}$.

was ranked by propensity score, and the patients were closely matched on the basis of this score. Short- and long-term outcomes of the patients were blinded during the matching process. Resulting matched patients with UACP $(n=246)$ and BACP $(n=246)$ were analyzed for differences in the selected early outcomes: intensive care unit stay, prolonged ventilation, reexploration for bleeding, acute renal failure requiring hemofiltration, TND and PND, and early mortality. All statistical analyses were performed with StatView, version 5.0, for Windows softwareQ2 (SAS Institute, Inc, Cary, NC).

\section{RESULTS}

As a result of the matching process, there was no difference among patients in both groups in terms of age, incidence of coronary artery disease, diabetes, chronic renal

TABLE 2. Demographics for propensity-matched patients undergoing aortic arch replacement during selective unilateral or bilateral antegrade cerebral perfusion and mild systemic hypothermic circulatory arrest

\begin{tabular}{|c|c|c|c|}
\hline Characteristic & UACP & BACP & $P$ value \\
\hline No. of patients & 246 & 246 & NA \\
\hline Age $(y \pm S D)$ & $66 \pm 9$ & $65 \pm 11$ & $>.99$ \\
\hline Male (N/\%) & $164(67 \%)$ & $156(63 \%)$ & .63 \\
\hline Acute type A dissection (N/\%) & 0 & 0 & NA \\
\hline Degenerative aneurysm (N/\%) & $246(100 \%)$ & $246(100 \%)$ & NA \\
\hline Coronary artery disease $(\mathrm{N} / \%)$ & $39(16 \%)$ & $42(17 \%)$ & .68 \\
\hline Diabetes $(\mathrm{N} / \%)$ & $20(8 \%)$ & $18(7 \%)$ & .65 \\
\hline Chronic renal failure $(\mathrm{N} / \%)$ & $13(5 \%)$ & $13(5 \%)$ & $>.99$ \\
\hline Previous stroke $(\mathrm{N} / \%)$ & $22(9 \%)$ & $20(8 \%)$ & .73 \\
\hline $\operatorname{COPD}(\mathrm{N} / \%)$ & $22(9 \%)$ & $26(11 \%)$ & .68 \\
\hline NYHA class & $2.1 \pm 1.2$ & $2.3 \pm 1.6$ & .77 \\
\hline Aortic valve stenosis (N/\%) & $23(9 \%)$ & $21(9 \%)$ & $>.99$ \\
\hline Aortic valve regurgitation $>2^{\circ}(\mathrm{N} / \%)$ & $56(21 \%)$ & $50(21 \%)$ & .88 \\
\hline Previous cardiac surgery $(\mathrm{N} / \%)$ & 0 & 0 & NA \\
\hline
\end{tabular}

$B A C P$, Bilateral antegrade cerebral perfusion; $C O P D$, chronic obstructive pulmonary disease; $N A$, not available; $N Y H A$, New York Heart Association; $S D$, standard deviation; $U A C P$, unilateral antegrade cerebral perfusion. failure, and history of stroke, which was present in 22 patients $(9 \%)$ in the UACP group and 20 patients $(8 \%)$ in the BACP group $(P \geq .65)$. CPB and selective ACP times were similar between the groups and averaged $164 \pm 41$ minutes and $41 \pm 19$ minutes, respectively, in the UACP group and $157 \pm 37$ minutes and $43 \pm 21$ minutes, respectively, in the BACP group $(P \geq .81)$. The extent of arch resection was not different between the groups: Total arch replacement was required in 91 patients $(37 \%)$ in the UACP group and 93 patients (38\%) in the BACP group, whereas the remaining patients received hemi-arch replacement $(P \geq .59)$. The lowest core temperature averaged $30^{\circ} \mathrm{C}$ $\pm 2{ }^{\circ} \mathrm{C}$ in both groups (Table 2 ).

Operative data and surgical procedures for propensitymatched patients undergoing aortic arch replacement with UACP or BACP are summarized in Table 3. Early morbidity and mortality are shown in Table 4. Mean intensive care unit stay averaged $2 \pm 7$ days in the UACP group and $3 \pm 11$ days in the BACP group and was not different between both groups $(P=.81)$. The incidence of prolonged ventilation was comparable between both groups and required in 12 patients $(5 \%)$ with UACP and 14 patients $(6 \%)$ with BACP, respectively $(P=.83)$. The incidence of reexploration for bleeding was similar between the groups $(7 \%$ in the UACP group and $9 \%$ in the BACP group), as was the rate of new-onset renal failure requiring temporary renal replacement therapy $(5 \%$ in the UACP group vs $4 \%$ in the BACP group) $(P \geq .52)$.

TND was observed in 12 patients $(5 \%)$ in the UACP group and 10 patients $(4 \%)$ in the BACP group $(P=.69)$. There was a trend toward a higher incidence of PND in the BACP group (14 patients, $6 \%$ ) compared with patients in the UACP group (5 patients, $2 \%)(P=.06)$. All PNDs were central in origin, and no paraplegia was observed in either group. Early mortality was comparable between the groups, being present in 9 patients $(4 \%)$ in the UACP group and 11 patients $(4 \%)$ in the BACP group $(P=.84)$.

The subgroup of patients with total arch replacement ( $\mathrm{n}=91$ in the UACP group vs $\mathrm{n}=93$ in the BACP group)

TABLE 3. Operative data and surgical procedures for propensitymatched patients undergoing aortic arch replacement during selective unilateral or bilateral antegrade cerebral perfusion and mild systemic hypothermic circulatory arrest

\begin{tabular}{lccc}
\hline \multicolumn{1}{c}{ Characteristic } & UACP & BACP & $\boldsymbol{P}$ value \\
\hline CPB time $(\min \pm \mathrm{SD})$ & $164 \pm 41$ & $157 \pm 37$ & .81 \\
Crossclamp time $(\min \pm \mathrm{SD})$ & $99 \pm 36$ & $95 \pm 41$ & .70 \\
Isolated ACP time $(\min \pm \mathrm{SD})$ & $41 \pm 19$ & $43 \pm 21$ & $>.99$ \\
Lowest core temperature $\left({ }^{\circ} \mathrm{C} \pm \mathrm{SD}\right)$ & $30 \pm 2$ & $30 \pm 2$ & $\mathrm{NA}$ \\
Hemi-arch replacement $(\mathrm{N} / \%)$ & $155(63 \%)$ & $153(62 \%)$ & .59 \\
Total arch replacement $(\mathrm{N} / \%)$ & $91(27 \%)$ & $93(28 \%)$ & .71 \\
Concomitant AVS $(\mathrm{N} / \%)$ & $79(32 \%)$ & $71(29 \%)$ & .69 \\
\hline
\end{tabular}

$A C P$, Antegrade cerebral perfusion; $A V S$, aortic valve surgery; $B A C P$, bilateral antegrade cerebral perfusion; $C P B$, cardiopulmonary bypass; $N A$, not available; $S D$, standard deviation; $U A C P$, unilateral antegrade cerebral perfusion. 
TABLE 4. Operative outcome of propensity-matched patients undergoing aortic arch replacement during selective unilateral or bilateral antegrade cerebral perfusion and mild systemic hypothermic circulatory arrest

\begin{tabular}{lccc}
\hline \multicolumn{1}{c}{ Characteristic } & UACP & BACP & $\boldsymbol{P}$ value \\
\hline ICU stay $(\mathrm{d} \pm \mathrm{SD})$ & $2 \pm 7$ & $3 \pm 11$ & .81 \\
Prolonged ventilation $(>7 \mathrm{~d})(\mathrm{N} / \%)$ & $12(5 \%)$ & $14(6 \%)$ & .83 \\
Reexploration for bleeding (N/\%) & $17(7 \%)$ & $22(9 \%)$ & .64 \\
ARF requiring hemofiltration (N/\%) & $13(5 \%)$ & $9(4 \%)$ & .52 \\
Temporary neurologic deficit (N/\%) & $12(5 \%)$ & $10(4 \%)$ & .69 \\
PND (N/\%) & $5(2 \%)$ & $14(6 \%)$ & .06 \\
Stroke/coma (N/\%) & $5(2 \%)$ & $14(6 \%)$ & \\
Paraplegia (N/\%) & 0 & 0 & \\
30-d mortality (N/\%) & $9(4 \%)$ & $11(4 \%)$ & .84 \\
\hline
\end{tabular}

$A R F$, Acute renal failure; $B A C P$, bilateral antegrade cerebral perfusion; $I C U$, intensive care unit; $P N D$, permanent neurologic deficit; $S D$, standard deviation; $U A C P$, unilateral antegrade cerebral perfusion.

and prolonged ACP times were further analyzed for outcomes. Mean ACP and CPB times in this subset of patients averaged $65 \pm 13$ minutes and $191 \pm 38$ minutes, respectively, in the UACP group and $67 \pm 18$ minutes and 184 \pm 31 minutes, respectively, in the BACP group $(P \geq .53)$. Most important, similar results regarding TND (6/91:7\% vs $5 / 93: 5 \% ; P=.7)$ and PND $(2 / 91: 3 \%$ vs $9 / 93: 9 \%$; $P=.06)$ rates were again observed when looking at $\mathrm{UACP}$ versus BACP in matched patients with total arch replacement only. Early mortality was no different between patients receiving UACP (6/91:7\%) and BACP (8/93:8\%) in this particular subgroup $(P=.63)$.

\section{DISCUSSION}

Neurologic dysfunction after aortic arch operations, either temporary or permanent, is a severe complication that strongly correlates with increased length of stay and mortality. ${ }^{15}$ Deep hypothermic circulatory arrest has been the mainstay of cerebral protection during operation on the aortic arch since its introduction. ${ }^{1}$ Apart from the drawbacks of deep hypothermic circulatory arrest, it does have certain limitations, especially when prolonged arrest times are required for arch repair. The introduction of ACP to accompany the circulatory arrest as a means to protect the brain during arch repair made deep hypothermia nonessential. ${ }^{5,6}$ In addition, several recent experimental reports provided evidence that cold cerebral perfusion can actually lead to neurologic injury because it, among other causes, negatively interferes with cerebral autoregulation. ${ }^{16,17}$

MSH preserves cerebral autoregulation and favors equal distribution of perfusion in the brain. ${ }^{18}$ The alpha-stat strategy for acid-base management on CPB just adds to it. ${ }^{19}$ During the last decade, several groups have reported improved results in urgent or elective aortic arch surgery when ACP was combined with $\mathrm{MSH} .^{7-9,20-23}$ The improved results in these reports are in greater part because of the extension of the safe period of cerebral protection, omitting deep hypothermic circulatory arrest, thus shortening the time of $\mathrm{CPB}$, all of them achievable only when ACP is combined with MSH.

Cannulation sites for UACP in conjunction with MSH include the right brachial artery, axillary artery, subclavian artery, innominate artery, or one of both carotid arteries. ${ }^{7-10}$ BACP is typically established with an additional cannula in the left common carotid artery. ${ }^{24,25}$ UACP is fast to establish, is simpler than BACP because no additional cannula is placed in the operating field, and avoids any manipulation on the ostia of the supra-aortic arteries, which are commonly involved with the arch pathology. At the same time, UACP has raised some criticism. The fear of putting the directly perfused hemisphere at increased risk of brain tissue edema because of cerebral hyperperfusion has been debated mostly by strong proponents of BACP.

In this study, we undertook an effort to determine the effectiveness of UACP versus BACP as brain-protection methods and their impact on early outcomes after aortic arch replacement using MSH. We included large cohorts with consecutive patients undergoing operation in 2 referral centers in Germany using similar perfusion and temperature management protocols. Patients with a type A dissection were excluded from further analysis because the presence of type A dissection alone can negatively affect preoperative neurologic status and postoperative neurologic outcomes. Overall, the rates of temporary or PNDs and early mortality were low and comparable between both groups, indicating a noninferiority of UACP compared with BACP. In addition, a trend toward a higher incidence of stroke, probably due to manipulation on the arch vessels, was observed in the BACP group.

Nonetheless, UACP represents a nonphysiologic circulation, relying on the completeness and competence of primary collateral pathway (circle of Willis), as well as secondary extracranial collateral networks. Various anatomic and angiographic studies reported variations of the normal morphology of the circle of Willis in as much as $50 \%$ of the patients being studied. ${ }^{26-28}$ Which of these variations, partial or complete, involving only 1 or simultaneously both of the communicating arteries, is relevant during surgery on the aortic arch has not been sufficiently studied and remains unclear. It also remains unclear whether such anatomic or pathologic abnormalities play an important role once both ipsilateral (right) carotid and vertebral arteries (and therefore extracranial collaterals) are being perfused. In a study on 99 patients undergoing open arch repair, Urbanski and colleagues ${ }^{29}$ assessed the completeness of the circle of Willis in all patients preoperatively with computed tomography angiography and found that the circle was complete in only 59 patients. The rest of the patients had partially (1-sided) or fully (2-sided) incomplete circle of Willis. Nevertheless, 
functional tests during carotid artery crossclamping and intraoperative cerebral monitoring, including transcranial Doppler, showed no pathology in any patient, concluding that the anatomic status of the circle of Willis assessed with cranial computed tomography angiography does not correlate with functional and intraoperative tests examining the cerebral cross-perfusion. Harrer and colleagues ${ }^{30}$ found a significant decrease in brain oxygen saturation when performing UACP with cold $\left(23^{\circ} \mathrm{C}\right)$ blood. The difference may be explained by the fact that the human brain loses its autoregulation in deep hypothermia. In general, the clinical impact of a decrease in cerebral oxygen saturation as estimated by NIRS monitoring has yet to be defined. In both centers, NIRS was routinely applied for neuromonitoring in the latter half of the series. In our experience, a decrease of NIRS measurements in the contralateral hemisphere during UACP to $70 \%$ of the baseline values was not associated with an impaired neurologic outcome.

Several reports have compared UACP with BACP in aortic arch operations. Olsson and Thelin ${ }^{24}$ compared small cohorts of patients (17 patients each) operated in deep hypothermia, rendering the study rather underpowered. Of note, $86 \%$ of all mortality was in the BACP group. Krahenbühl and coworkers ${ }^{25}$ included more patients $(\mathrm{n}=280)$ and did not find any difference regarding early mortality, temporary or permanent neurologic morbidity, and length of intensive care unit stay. However, they did find improved quality of life in those receiving BACP. The largest comparative study until now came from the German Registry for Acute Aortic Dissection Type A and included 1081 patients operated for type A dissection in 44 centers, ${ }^{31}$ which found that early outcomes regarding mortality and neurologic morbidity with UACP and BACP were equivalent. The same conclusion was reached in the only meta-analysis published on this issue to date. Angeloni and colleagues, ${ }^{32}$ in an article published in the Journal, analyzed 28 studies $(>5100$ patients) reporting on mortality and neurologic morbidity in aortic surgery requiring circulatory arrest and ACP. They also found no difference in early outcomes between $\mathrm{UACP}$ and BACP.

\section{Study Limitations}

Despite the process of propensity score matching and the fact that we included all consecutive patients operated in the study period and excluded all acute type A dissections, the main limitation of this study remains is its retrospective nature. Of note, there is no prospective randomized study on this issue to date.

\section{CONCLUSIONS}

We used a propensity-matched analysis in a large cohort of patients undergoing elective aortic arch surgery and found that using UACP in a pressure-controlled manner during MSH provides a safe and noninferior protection strategy, associated with similar morbidity and mortality in comparison with BACP, even if total arch replacement is required.

\section{References}

1. Griepp RB, Stinson EB, Hollingsworth JF, Buehler D. Prosthetic replacement of the aortic arch. J Thorac Cardiovasc Surg. 1975;70:1051-63.

2. Mexrow CK, Sadeghi AM, Gandsas A, Shiang HH, Levy D, Green R, et al. Cerebral blood flow and metabolism in hypothermic circulatory arrest. Ann Thorac Surg. 1992;54:609-15.

3. Strauch JT, Spielvogel D, Haldenwang PL, Haldenwang PL, Zhang N, Weisz D, et al. Impact of hypothermic selective cerebral perfusion compared with hypothermic cardiopulmonary bypass on cerebral hemodynamics and metabolism. Eur J Cardiothorac Surg. 2003;24:807-16.

4. Crittenden MD, Roberts CS, Rosa L, Vatsia SK, Katz D, Clark RE, et al. Brain protection during circulatory arrest. Ann Thorac Surg. 1991;51:942-7.

5. Bachet J, Guilmet D, Goudot B, Termignon JL, Teodori G, Dreyfus G, et al. Cold cerebroplegia: a new technique of cerebral protection during operations on the transverse aortic arch. J Thorac Cardiovasc Surg. 1991;102:85-94.

6. Kazui T, Kimura N, Yamada O, Komatsu S. Surgical outcome of aortic arch aneurysm using selective antegrade perfusion. Ann Thorac Surg. 1994;57: 904-11.

7. Tasdemir O, Saritas A, Kucuker S, Ozatik MA, Sener E. Aortic arch repair with right brachial artery perfusion. Ann Thorac Surg. 2002;73:1837-42.

8. Urbanski PP. Cannulation of the left common carotid artery for proximal aortic repair. J Thorac Cardiovasc Surg. 2003;126:887-8.

9. Zierer A, Aybek T, Risteski P, Dogan S, Wimmer-Greinecker G, Moritz A. Moderate hypothermia $\left(30^{\circ} \mathrm{C}\right)$ for surgery of acute type A aortic dissection. Thorac Cardiovasc Surg. 2005;53:74-9.

10. Neri E, Massetti M, Capannini G, Carone E, Tucci E, Diciolla F, et al. Axillary artery cannulation in type A aortic dissection operations. J Thorac Cardiovasc Surg. 1999;118:324-9.

11. Zierer A, Detho F, Dzemali O, Aybek T, Moritz A, Bakhtiary F. Antegrade cerebral perfusion with mild hypothermia for aortic arch replacement: single-center experience in 245 consecutive patients. Ann Thorac Surg. 2011; 91:1868-73.

12. Zierer A, El-Sayed Ahmad A, Papadopoulos N, Moritz A, Diegeler A, Urbanski PP. Selective antegrade cerebral perfusion and mild $\left(28-30^{\circ} \mathrm{C}\right)$ systemic hypothermic circulatory arrest for aortic arch replacement: results from 1002 patients. J Thorac Cardiovasc Surg. 2012;144:1042-9.

13. Urbanski PP, Lenos A, Lindemann Y, Weigang E, Zacher M, Diegeler A. Carotid artery cannulation in aortic surgery. J Thorac Cardiovasc Surg. 2006;132: $1398-403$.

14. Urbanski PP, Lenos A, Bougioukakis P, Neophytou I, Zacher M, Diegeler A Mild-to-moderate hypothermia in aortic arch surgery using circulatory arrest: a change of paradigm? Eur J Cardiothorac Surg. 2012;41:185-91.

15. Hagl C, Ergin MA, Galla JD, Lansman SL, McCullough JN, Spielvogel D, et al. Neurologic outcome after ascending aorta-aortic arch operations: effect of brain protection technique in high risk patients. J Thorac Cardiovasc Surg. 2001;121: 1107-21.

16. Strauch JT, Haldenwand PL, Müllem K, Schmalz M, Liakopoulos O, Christ H, et al. Temperature depending cerebral blood flow for isolated regions of the brain during selective cerebral perfusion in pigs. Ann Thorac Surg. 2009;88:1506-13.

17. Haldenwand PL, Strauch JT, Müllem K, Liakopoulos O, Reiter H, Lehmacher W, et al. Impact of pressure management during hypothermic selective cerebral perfusion on cerebral hemodynamics and metabolisms in pigs. Thorac Cardiovasc Surg. 2009;56(Suppl 1):S24-33.

18. Georgiadis D, Schwarz S, Evans DH, Schwab S, Baumgartner RW. Cerebral au toregulation under moderate hypothermia in patients with acute stroke. Stroke 2002;33:3026-9.

19. Patel RL, Turtle MR, Chambers DJ, James DN, Newman S, Venn GE. Alpha-stat acid- base regulation during cardiopulmonary bypass improves neurophysiologic outcomes in patients undergoing coronary artery bypass grafting. J Thorac Cardiovasc Surg. 1996;111:1267-79.

20. Panos A, Murith N, Bednarkiewicz M, Khatchatourov G. Axillary cerebral perfusion for arch surgery in acute type A dissection under moderate hypothermia. Eur J Cardiothorac Surg. 2006;29:1036-40. 
21. Numata S, Thomson DS, Seah P, Singh T. Simplified cerebral protection using unilateral antegrade cerebral perfusion and moderate hypothermic circulatory arrest. Heart Lung Circ. 2009;18:334-6.

22. Saritas A, Kervan U, Vural KM, Kucuker SA, Yavas S, Birincioglu LC. Visceral protection during moderately hypothermic selective antegrade cerebral perfusion through right brachial artery. Eur J Cardiothorac Surg. 2010;37:669-76.

23. Leshnower BG, Myung RJ, Kilgo PD, Vassiliades TA, Vega JD, Thourani VH, et al. Moderate hypothermia and unilateral selective antegrade cerebral perfusion: a contemporary cerebral perfusion strategy for aortic arch surgery. Ann Thorac Surg. 2010;90:547-54.

24. Olsson C, Thelin S. Antegrade cerebral perfusion with simplified technique: unilateral versuss bilateral perfusion. Ann Thorac Surg. 2006;81:868-74.

25. Krahenbühl ES, Clement M, Reineke D, Czerny M, Stalder M, Aymard T, et al. Antegrade cerebral protection in thoracic aortic surgery: lessons from the past decade. Eur J Cardiothorac Surg. 2010;38:46-51.

26. Macchi C, Catini C, Federico C, Gulisano M, Pacini P, Cecchi F, et al. Magnetic resonance angiographic evaluation of circulusarteriosuscerebri (circle of Willis): a morphologic study in 100 human healthy subjects. Ital J Anat Embryol. 1996; 101:115-23.

27. Papantchev V, Hristov S, Todorova D, Naydenov E, Paloff A, Nikolov D, et al. Some variations of the circle of Willis, important for cerebral protection in aortic surgery - a study in Eastern Europeans. Eur J Cardiothorac Surg. 2007;31:982-9.

28. Merkkola P, Tulia H, Ronkainen A, Soppi V, Oksala A, Koivisto T, et al. Incomplete circle of Willis and right axillary artery perfusion. Ann Thorac Surg. 2006; 82:74-80.

29. Urbanski PP, Lenos A, Blume JC, Ziegler V, Griewing B, Schmitt R, et al. Does anatomical completeness of the circle of Willis correlate with sufficient crossperfusion during unilateral cerebral perfusion. Eur J Cardiothorac Surg. 2008; $33: 402-8$.

30. Harrer M, Waldenberger FR, Weiss G, Folkmann S, Gorlitzer M, Moidl R, et al. Aortic arch surgery using bilateral antegrade selective cerebral perfusion in combination with near-infrared spectroscopy. Eur J Cardiothorac Surg. 2010;38: 561-7.

31. Krüger T, Weigang E, Hoffmann I, Blettner M, Aebert H. Cerebral protection during surgery for acute aortic dissection type A: results of the German Registry for Acute Aortic Dissection Type A (GERAADA). Circulation. 2011;124: 434-43.

32. Angeloni E, Benedetto U, Takkenberg JJM, Stigliano I, Roscitano A, Melina G, et al. Unilateral versus bilateral antegrade cerebral protection during circulatory arrest in aortic surgery: a meta-analysis of 5100 patients. J Thorac Cardiovasc Surg 2012;334-7.

\section{Discussion}

Dr E. Raanani (Tel Hashomer, Israel). The issue of what is the best method to protect the brain during aortic arch surgery has been debatable for several decades. Many questions are still not answered. Hypothermia combined with ACP emerged as the best method in cases where prolonged systemic circulatory arrest times are needed.

Because anatomic and imaging studies showed that up to $50 \%$ of adult population have variations and interruption of the brain's circle of Willis, it seems logical that BACP, meaning perfusion to both carotid arteries, would be the more physiologic method to protect the brain. However, several groups reported on using only moderate hypothermia and only UACP as a method of choice with good clinical outcomes.

To better clarify this debate, Dr Zierer and colleagues retrospectively collected their large experience of aortic arch surgery from 2 medical centers, Frankfurt and Bad Neustadt. They propensitymatched 2 groups with 246 patients each. UACP was used in one group, and BACP was used in the other group. Both used MSH. NIRS was used for brain monitoring. Approximately $30 \%$ of the patients in each group underwent full arch replacement. The rest underwent partial arch.
The main findings in this study are that there are no differences in early mortality between the groups and a tendency with a $P$ value of .06 for more permanent neurologic events in the BACP group. I have 4 questions.

In the study, you excluded patients who were converted from UACP to BACP during surgery. You did not provide the numbers and their outcomes. Can you provide us with this information? And if they were included in an "intention to treat" analysis, would it affect the results of this study?

Dr Zierer. There were only 6 patients who were converted from UACP to BACP for the entire series, and we thought that we would not include them because they might be a selection of more complicated cases and would affect the findings or negatively influence the outcome of BACP. These patients were converted because of the decrease in the NIRS measurements and a decrease in the contralateral radial artery pressure, and then we switched to BACP. But all 6 patients recovered well. There was no permanent and only 1 transient neurologic deficit.

Dr Raanani. There is a significant difference in technique of arch vessel cannulation between the 2 centers, with Frankfurt using cannulation of the axillary artery and snaring of the proximal part of the carotid arteries, and Bad Neustadt directly perfusing the arch vessels using an 8-mm or 10-mm Dacron extension with crossclamping of the arch vessel at the origin. Moreover, Frankfurt uses implantation of the arch vessels en bloc, and Bad Neustadt uses separate anastomoses. It seems that the Bad Neustadt technique involves more manipulation of the arch vessels that may contribute to ipsilateral embolization and brain ischemia. Did you check your results according to the different centers?

Dr Zierer. Regarding permanent and transient neurologic complications, there was no difference among the 2 centers. But you're certainly right, the different cannulation technique applied is the main difference between the 2 groups, and this is also the reason why they had a higher ACP flow in Bad Neustadt because they also perfuse the right arm during ACP.

Dr Raanani. Can you elaborate on how you decided to perfuse the brain? Was it according to preoperative imaging of the brain circulation or surgical complexity? If yes, do you have any potential bias that such patients who underwent BACP had more interrupted brain circulation to start with or more complex arch pathologies?

Dr Zierer. There is a different approach to this topic in the 2 centers. In Frankfurt, until 2005, we routinely performed UACP and then switched to BACP between 2005 and 2010. Because of these recent data, we switch back more frequently to UACP in more recent years.

If we are planning a procedure now, also an elective total arch replacement, we would start with UACP. But we always have a Y-shaped arterial line connected to the arterial pump line ready in case the NIRS measurements decrease to less than $70 \%$ of the baseline value so that we can easily switch to BACP if desired.

If we take a look at the subgroup of patients with total arch replacement only, they had an ACP time of approximately 60 minutes. Despite the limited number of patients with total arch replacement it seems that UACP can be safely performed at least up to 60 minutes.

Dr Raanani. So it means that even if in the preoperative imaging you see that there is an interruption of the circle of Willis, you would start with UACP? 
Dr Zierer. In the current phase, we would do so, yes, because various studies also from Dr Urbanski in Bad Neustadt showed that the completeness or incompleteness of circle of Willis in patients undergoing surgery with UACP did not correlate with intraoperative NIRS findings or postoperative neurologic outcomes.

Dr Raanani. This brings me to my last question. In view of these study results, are there any special situations where you recommend BACP or do you practice only UACP now, which you answered already. And in special cases, would you recommend other methods, such as more deep hypothermia for instance or not?

Dr Zierer. Regarding temperature management, we would only cool down further in patients with complex acute type A dissections. If the right axillary artery is dissected as well, then of course we would have to switch to femoral artery cannulation. But including type A dissections in the last 5 years, we hardly ever cooled down to less than $26^{\circ} \mathrm{C}$. I would say $95 \%$ of patients undergoing aortic arch surgery had a lowest core temperature of $27^{\circ} \mathrm{C}$ to $28^{\circ} \mathrm{C}$. For elective cases, we would start with UACP and keep an eye on the NIRS measurements and the contralateral radial artery pressure.

Dr M. Moon (St Louis, Mo). Did you have any problems with direct cannulation of the axillary artery? We generally use a graft whether it is the axillary, carotid, or innominate artery.

Dr Zierer. We have not experienced problems with direct axillary artery cannulation in more than 500 patients now. Early in our experience, there were 2 patients with transient plexus lesions, but they recovered before discharge. Other than that, we did not experience any problems.

Dr Moon. What kind of cannula tip do you use? Do you get any flow down the right arm during that period of time?

Dr Zierer. No. The right arm is occluded during this time, which is no problem at all in our experience.

Dr Moon. What did you speculate was the cause of the stroke on the left side, was it embolization from manipulation from the catheters?

Dr Zierer. Probably. That's what it looks like, yes. This might be surgeon dependent because our chief in Frankfurt preferred BACP and asked me to go back and take a close look at all patients who had a stroke after BACP, and, in fact, he did not have a single stroke. So everyone manipulating on the arch vessels for establishment of BACP has to be aware of the potential risk of cerebral embolization.

Dr Moon. Well, we use UACP. We don't use the balloons from the inside. We dissect the arteries from the outside and clamp them with a standard vascular clamp, and that avoids any potential for error or debris to go up that carotid while you're doing the perfusion.

Dr Zierer. We also use the balloon only for additional perfusion in case of BACP. If you occlude it during UACP, we just clamp it from the outside as well.

Dr Moon. Yes, clamp it with a vascular clamp.

Dr A. Pochettino (Rochester, Minn). The question you pose in your article is worthwhile; however, I am not sure that you have been able to answer the issue, that is, two thirds of your cases were hemi-arches, and one could argue that worrying about $\mathrm{UACP}$ or BACP is probably irrelevant for an operation that should last less than 20 minutes.

The only patients in whom it may be of relevance to look at this question are those undergoing total arch replacement, but the number of total arches you're looking at here are few. Therefore, it's hard to draw any conclusion other than the more you manipulate a vessel, the more likely you're going to have embolic events. I applaud you for asking this difficult question, but I think you should focus on the total arch replacement only. Of course, manipulation is to be guarded against because most strokes are embolic events.

Dr Zierer. It is certainly true that total arch replacements are most important and interesting. I agree that the number of patients in this subgroup is limited, but we tried to strengthen the data by implementing propensity analysis. Also, I'm not sure if you're aware of a recent publication in the Journal, a meta-analysis on 5100 patients including one third of total arch replacements, and they also saw no difference regarding neurologic outcome with UACP versus BACP.

The longer the ACP duration is, the more important a potential benefit of BACP might be. So far, I think there is no evidence that $\mathrm{BACP}$ is really better.

Dr M. Moon (St Louis, Mo). What's your method now, then? Are you only doing UACP?

Dr Zierer. At this time, we start with UACP if a full-arch replacement is planned. We would switch to BACP only if it takes more than 60 minutes or there is any problem with the NIRS measurements or the radial artery pressure on the left side.

Dr Moon. Even the professor?

Dr Zierer. Even the professor.

Dr M. Deja (Katowice, Poland). You showed that you are actually using the blood pressure on the infusion site as the guidance for the flow. I understand, $80 \mathrm{~mm} \mathrm{Hg}$, yes? What flow does it correspond to actually? What are the flows you use in the unilateral infusion? I wonder whether you ever observed any unilateral edema in the brain at least transiently after the surgery because of the unilateral, let's say, overflow or excessive infusion.

Dr Zierer. In both centers, we use a rather high perfusion pressure of $80 \mathrm{~mm} \mathrm{Hg}$, which allows an ACP flow of 1.1 to $1.2 \mathrm{~L} / \mathrm{min}$ with direct axillary artery cannulation as applied in Frankfurt. In Bad Neustadt, because you have the additional perfusion of the right arm, it is even higher (1.5-1.6 L/min). We did not see signs of hyperperfusion in both contributing units.

Dr A. Laudito (Duluth, Minn). Would you share your experience with cannulation, through a graft, of the brachiocephalic artery.

Dr Zierer. In Frankfurt, we have little experience with this approach. We only choose this cannulation site if there are problems with the axillary artery. However, in the limited number of patients we used it, it worked out fine. 